\title{
WHITE DWARF MODELS OF SUPERNOVAE AND CATACLYSMIC VARIABLES
}

\author{
Ken'ichi Nomoto and Masa-aki Hashimoto
}

Department of Physics, Brookhaven National Laboratory

Upton, NY 11973, U.S.A.

on leave from the Department of Earth Science and Astronomy

College of Arts and Sciences, University of Tokyo

Meguro-ku, Tokyo 153, Japan

to appear in the Proceedings of IAU Colloquium 93

Cataclysmic Variables

Bamberg, F.R.G., June 16 - 19, 1986

ed. H. Drechsel, Y. Kondo and J. Rahe (D. Reidel)

\section{DISCLAIMER}

This report was prepared as an account of work sponsored by an agency of the United States Government. Neither the United States Government nor any agency thereof, nor any of their employees, makes any warranty, express or implied, or assumes any legal liability or responsibility for the accuracy, completeness, or usefulness of any information, apparatus, product, or process disclosed, or represents that its use would not infringe privately owned rights. Reference herein to any specific commercial product, process, or service by trade name, trademark, manufacturer, or otherwise does not necessarily constitute or imply its endorsement, recommendation, or favoring by the United States Government or any agency thereof. The views and opinions of authors expressed herein do not necessarily state or reflect those of the United States Government or any agency thereof. 
WHITE DWARF MODELS OF SUPERNOVAE AND CATACLYSMIC VARIABLES

\section{Ken'ichi Nomoto and Masa-aki Hashimoto}

Physics Department, Brookhaven National Leboratory; on leave from the Department of Earth Science and Astronomy, University of Tokyo

ABSTRACT: If the accreting white dwarf increases its mass to the Chandrasekhar mass, it will either explode as a Type I supernova or collapse to form a neutron mass, In fact, there is a good agreement between the exploding white dwarf model star. In fact, there is a good agreenent between the exploding white dwarf model
for Type I supernovae and abservations. We describe various types of evolution of accreting white dwarfs as a function of binary parameters (i.e, composition, mass, and age of the white dwarf, its companion star, and mass accretion rate), and discuss the conditions for the precursors of exploding or collapsing white dwarfs, and their relevance to cataclysmic variables. Particular attention is given to helium star cataclysmics which might be the precursors of some Type I supernovar of ult ull dwarfs, and discuss the composition structure and their relevance to the model for neon novae.

\section{INTRODUCTION}

The final fate of accreting white diarfs will be either therinonuclear explosion or collapse, if the white dwarf mass grows to the Chandrasek har mass. Though the exact precursor systens are not know yet, very good ngreement between the exploding white dwarf models (carbon defingration model) and the observed features of Type la supernovae (Nomoto 1986a; Woosley and Weaver 1986b) suggests that some accreting white $d$ warts actually increase their mass to the Chnndrasekhar inoss. For Type Ib supernovae, though currently most popular models are explosions of WolfRayet stars, the maximum light spectrum might better be explained by off-center explosions of white dwarfs. Furthermore, recent observations of several interesting binary systems, low mass X-ray binaries, QPOs, and binary radio pulsars have ing binary systems, low mass X-ray binaries, QPOs, and binary radio pulsars have suggested that in these syatems a neutron star has formed from accretion-induced
collapse of a white dwarf (van den Heuvel 1984; Taam and van den Heuvel 1986).

These variations in the final fate of accreting white dwarfs originate from the differences in the parameters of the binary system, namely, composition, mass, and age of the white dwarf, its companion star, and mass accretion rate. It is interesting to look into whether some cataclysmic variables meet the conditions for the precursors of exploding or collapsing white dwarfs. The maso accretion rate is a particularly useful parameter for this purpose.

In 52, we discuss how the evolution of accreting white dwarfs dependa on the binary parameters. In $\$ \mathbf{3}-\mathbf{g 4}$, theoretical models of explosion or collapse of white dwarfa are described and compared with the observations of Type le and th supernovee. In 55 , we discuss the evolution of helium atar eataclysmics, where the mase donor is not a hydrogen main-sequence star but a helium main-sequence otar. These aystems might be precursors of some Type 1 supernove or ultrashort period X-ray binariea. Finally, in So, we present a new evolutionary calculation to show the formation of $\mathrm{O}+\mathrm{Ne}+\mathrm{Mg}$ white dwarfs and their composition, because recently discovered neon novac raised new interest in the $\mathrm{O}+\mathrm{Ne}+\mathrm{Mg}$ white dwarls.

\section{THE FATE OF WHITE DWARFS AS A FUNCTION OF MASS AND} ACCRETION RATE

\subsection{Heating of Accreting Whiie Dwarfs}

Isolated white dwarfs are simply cooling stars that eventually end up as dark matter. In binary systems they evolve differently because mas accretion from their companion provides gravitational energy that rejuvenates them. The grevitational energy released at the accretion shock near the stellar surface is radiated awey and does not heat the white dwarf interior. However, the compression of the interior by the accreted matter releases additional gravitational errergy. Some of this energy goes into thermal energy (compressional heating) and the rest is transported to the surface and radiated away (radiative conling). Therefore, the interior tempera ture is determined by the balance between heating and cooling and, thus, strongly depends on the mass accretion rate, $\dot{M}$.

The actunl growth rate of the white dwarf mass, $\dot{M}_{e} n$, may be smaller then the mass aceretion rale, $\dot{M}$, because some fraction of the accreted matter might be lost as a result of hydrogen or helium shell Hashes as discussed below. However, com. pressional heating is still determined by $\dot{M}$, not by $\dot{M}_{e} n$, becausc the compression rate of natter at a Lagrangian shell of $\boldsymbol{M}_{\boldsymbol{r}}$ is devided into two terms as

$\lambda_{0} \equiv d \ln \rho / d t=\lambda_{0}^{(M)}+\lambda_{p}^{(n)}$.

$\lambda_{\rho}^{(M)}=(\partial \ln \rho / \partial \ln M)_{\mathrm{v}}\left(\dot{M}_{\mathrm{en}} / M\right)$,

$\lambda_{\rho}^{(q)}=-(\partial \ln \rho / \partial \ln q)_{M}(\dot{M} / M)$,

where $q \equiv M_{r} / M$ is the mass fraction, and heating near the surface is determined by $\lambda_{\rho}^{(q)} \propto \dot{M}$, not by $\lambda_{\rho}^{(M)} \propto \dot{M}_{\text {ent }}$, as scen $\lambda_{p}^{(j)} \propto M$, not by $\lambda_{p} \propto M_{e n}$, as seen
from Fig. 1 which shows the cose of $M=$ $1.28 M_{\odot}$ and $\dot{M}=7 \times 10^{-10} M_{\odot} y^{-1}$ (sce Nomoto 1082a for details).

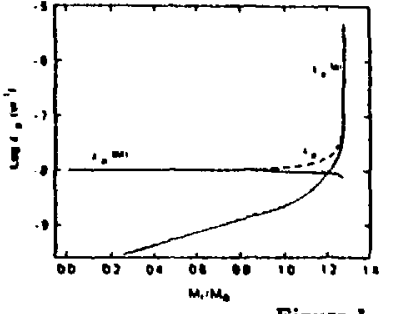

Figure 1 


\subsection{Accretion of Hydrogen-rich Matter and Hydrogen Flash}

First let us discuss the case where the hydrogen-rich matter accretes on the white dwarf. When a certain amount of moss, $\Delta M_{11}$, is accuinulated, hydrogen shell burning is ignited. $\Delta M_{\mathrm{H}}$, which is critical for the subsequent evolution, is determined by $M$ and $M$ as seen in Fig. 2 (Nariai 8nd Nomoto 1979; Nomoto 1982a). For $M<$ $0.4 \dot{M}_{R G}$ where $\dot{M}_{\mathrm{RG}}=8.5 \times 10^{-7}\left(M / M_{\odot}-0.52\right) \Lambda_{\odot} \mathrm{yr}^{-1}$, the hydrogen shell burning is unstable to $n$ flash. The flash gives rise to an expansion of the accreted envelope, a part of which is lost from the system. For larger $M$ and $\Delta M_{\mathrm{H}}$ (i.e., sinaller $\dot{M})$, the flash is stronger and causes larger mass loss. Therefore the white dwarf mass $M$ can hardly increase for $\dot{M}<10^{-8} M_{\odot} \mathrm{yr}^{-1}$, especially for nova-like explosion (Fujimoto and Taam 1982; MacDonald 1983, 1984).

2.3 Accretion of Heliurn and Helium Flash

On the other hand, a large portion of $\Delta M_{\mathrm{n}}$ ean be processed into helium for the accretion as rapid as $\dot{M} \sim 10^{-8}-10^{-6} M_{\odot} \mathrm{yr}^{-1}$. Then a helium layer is gradually built up on the $\mathrm{C}+\mathrm{O}$ white dwarf. This process is equivalent to the accretion of helium from a heliurn star (helium dwarf or helium star cataclysnics; see 55). Again a helium flash is ignited when a certain amount of helium, $\Delta M_{\text {IIe }}$ is nccumulated. $\Delta M M_{\mathrm{He}}$ is a function of $\dot{A}$ and mass of underlying $\mathrm{C}+\mathrm{O}$ core $M_{\mathrm{CO}}$ as given in $\Delta M_{\text {He }}$ is a function of $M$ and a mass of underlying $C+O$ core $M_{C O}$ as given in
Fig. 3 (Kawai et al. 1986). The flash is stronger for larger $M$ and $\Delta M_{\text {lile (i.e., }}$ lower $\dot{M}$ ) because of higher ignition density. It grows even into a helum deconation for $\dot{M}<\dot{M}_{\text {det }} \sim 4 \times 10^{-8}$ (Nornoto 1982b; Woosley et al. 1980) as indicated by the dashed line in Fig. 3 (see $\$ 3.2$ - $\$ 3.3$ ).
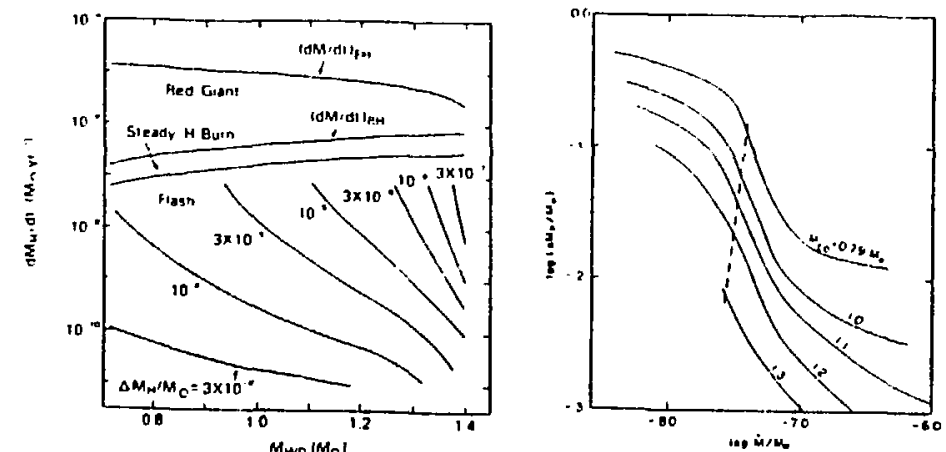

Figure 2 (left): The mass of accreted hydrogen-rich envelope, $\Delta M_{11}$, at the ignition of hydrogen flash as a function of $M$ and $M$.

Figure 3 (right): The mass of accreted helinm envelofe, $\Delta M_{\mathrm{e}}$, at the ignition of a helium flash as a function of $\dot{M}$ and the mass of underlying $\mathrm{C}+\mathrm{O}$ core, $M_{\mathrm{CO}}$
For higher $\dot{M}$, the helium flash is mild and will increase the $\mathrm{C}+\mathrm{O}$ eore mass (Tuem 1980; Fujimoto and Sugimoto 1982). Eventually earbon ignition ocrurs at the center and induces a carbon deflogration (\$3.1). The above scenario holds also for $\mathrm{O}+\mathrm{Ne}+\mathrm{Mg}_{\mathrm{g}}$ white dwarfs.

2.4 Off-Center Flash in Merging Double White Dwarls (CO - CO, Ile - He Pairs)

When two white dwarfs stant to merge owing to gravitational wave radiation (Iben and Tutukov 1984: Webbink 1984), rapid mass transfer from the less massive white dwarf is expected. In spherical accretion models for $\mathrm{C}+\mathrm{O}-\mathrm{C}+\mathrm{O}$ pair (Nomoto and Iben 1985), acesetion faster than $2.7 \times 10^{-6} M_{\odot} \mathrm{yr}^{-1}$ ignites a mild off-reiter carbon flash at $M_{r}=M_{r, i g}$ when the white dwarf mass reaches $M$, given in Fip. 1 as a fuistion of $\dot{M}$ (Kawai et al. 1980 , where $\Delta M_{e}=M_{0}-M_{r, i z}$ ). The carbon burning front, then, propagates inward all the why to the center and converts the $\mathrm{C}+\mathrm{O}$ white $\mathrm{dwar}$ into an $\mathrm{O}+\mathrm{Ne}+\mathrm{Mg}$ white dwarf (Snio and Nomoto 1985; Wonslry and Weaver 1980a).

For a lle - lle dwarf pair, accretion faster than $2 \times 10^{-8} M_{\odot} \mathrm{yr}^{-1}$ ignites a mild off-center helium flash (Nornoto and Sugimote 1977). The helium burning front also propagates to the center (Fig. 5; Saio and Nonoto 1986). There is a differenre from the $\mathrm{C}+\mathrm{O}-\mathrm{C}+\mathrm{O}$ case, however. The white dwart changes into helium mainsequence star, not directiy into a $\mathrm{C}+\mathrm{O}$ white dwarl, because only 10 percent of helium burns during the propapation. After lelium is exhausted, the star changes into a $\mathrm{C}+\mathrm{O}$ white dwarf
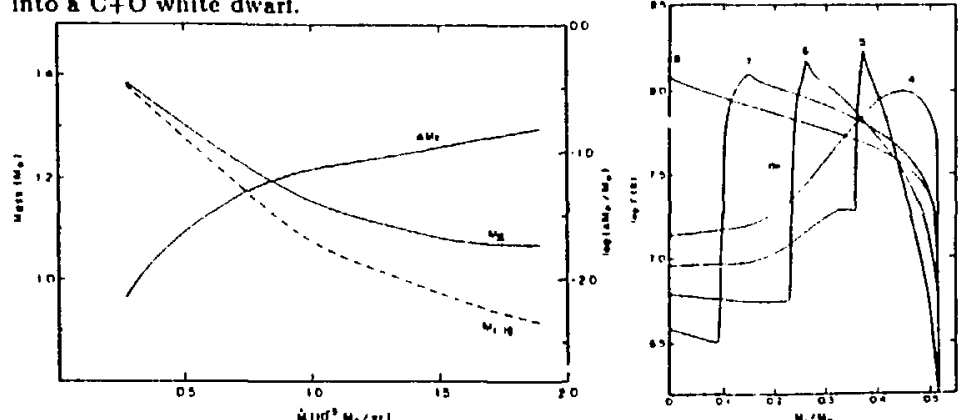

e 4 (left): The white dwarf mass (M) the location of the carbon burning shell $\left(M_{\mathrm{r}, \mathrm{ig}}\right)$, and $\Delta M_{\mathrm{e}}=M_{\mathrm{v}}-M_{\mathrm{t}, \mathrm{ig}}$ at the carbon ignition. These values depent only on $\dot{M}$. For $\dot{M}>2.7 \times 10^{-6} M_{\odot} \mathrm{yt}^{-1}$, central ignition occurs.

Figure 5 (right): Change in the temperature profile during the propagation of the heling $1 \times 10^{-7} M_{\odot} y^{-1}$. At stage 8 , the front reaches the center and the star becones a helium mnin-sequence star. 
2.5 Long Term Evolution leading to Supenova Explosion or Collapse

When the underlying $\mathrm{C}+\mathrm{O}$ core grows gradunlly, the white dwarf evolves in the following way nud its final fate depends critically on the initial mass $M_{C O}$ (i.e., the mass at the onset of nccretion) ns well as $\dot{M}$. Compression first heats up $n$ layer near the surface because of the stmall pressure senle height there (sre $\lambda_{r}^{(r)}$ in Fig. 1). Later, heat diffuses inward. The diffusion timescese depends on $M$ nnd is small for larger if because of the inge hent flux and steep tempernture gradient generated by rapid accretion. For exnmple, the time it takes the heat wave to reache the central region is about $2 \times 10^{5}$ yr for $\dot{M} \sim 10^{-6} M_{\odot} \mathrm{yr}^{-1}$ and $5 \times$ $10^{6}$ yr for $\dot{M} \sim 1 \times 10^{-8} M_{\odot} y_{r^{-1}}$. Therefore, if the initial mass of the white dwarf, $M_{\mathrm{CO}}$, is smaller than $1.2 M_{\odot}$ (this value is larger if $\left.M_{\mathrm{r}}<M\right)$, the entropy in the center inc 政 white dwarf is sufficiently massive and cold at the onset of nccretion, the rentra region is compressed only adinbatically and thus is cold when enrbon burning is ignited in the center. In the latter case, the ignition density is as high as $10^{10} \mathrm{R}$ $\mathrm{cm}^{-3}$ (e.g., Isern et al. 1083).

Acrordingly, the ultimnte fate of accreting $\mathrm{C}+\mathrm{O}$ white $\mathrm{dw}$ arfs depends on $\mathrm{Mi}$ and the inintial mass of the white $d w a r f M_{C O}$, ns summarized in Fig. 6 . $M$ denctes the growth rale of the while dwerf mass irrespective of the composition of the accretime matter. A similar diagram for the O+Ne+Ne white dwarfs is shown in Fin 7 Det \$3- $\$ 4$.

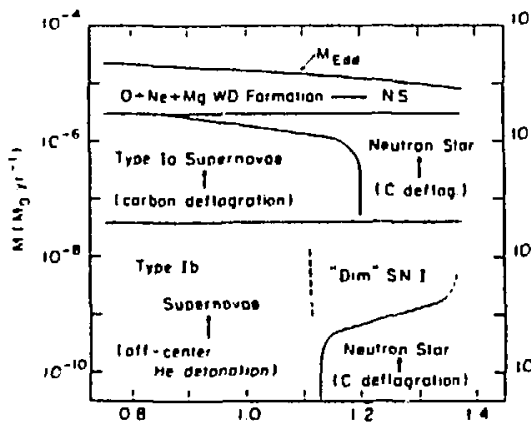

$M_{c-0}\left(M_{0}\right)$

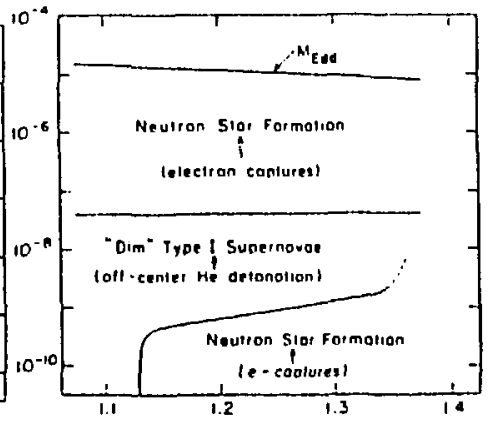

Monomotimo
Figure 6 (left): The final fite of acereting $C+1 O$ white dwarfs expected for their initial mass $M$ and necretion rate $\dot{A}$.

Figure 7 (right): Same as Fig. 6 hut for $\mathrm{O}+\mathrm{Ne}+\mathrm{Mg}_{\mathrm{g}}$ white dwarls.
2.6 Candidates of the Supernova or Collapse Precursors

As discussed in $\$ 2.2$, rather high arcretion rates $\left.(\dot{\Lambda})>10^{-8} M_{\odot} y^{-1}\right)$ are required in order for the acereting white dwhrf to berome a Type 1 supernown or neutron star because nova explosions and helium detonations should be nvoided. Possibel binnry systems to realize such accretion rntes include:

(1) Case $A$ mnss transfer on the thermal timescale from a main-sequenes star of $\sim$

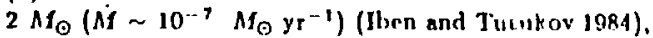

(2) Minss transfer on the nuclear tinicscale from a sulginnt of $\sim 0.8 \mathrm{M}{ }_{\mathrm{O}}(\mathrm{Mi} \sim 10$, 10 $10^{-A} M_{\odot} \mathrm{yr}^{-1}$ ) (Weblink et al. 1083).

(3) Accretion of helium from a helium emain-sequener star on the timescale of gravitational wave radintion $\left(\sim 2.0 \times 10^{-8} M_{\odot} y^{-1}\right.$ ) (see 65 ; Snvonijo ot al. 1880

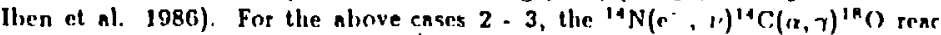
tion would be important to lower $M_{\text {det }}$ from $4 \times 10^{\circ}$ to $1 \times 10^{8} M_{O}$ yr (IInshimoto et al. 1980).

(4) Double $\mathrm{C}+\mathrm{O}$ white dwarfs if $\dot{M}<2.7 \times 10^{-6} M_{0}$ yr 1 or $1 /$ nom-splierient efferts prevents off-center carhon hurning from igniting or propagnting. If $M$ is higher, collapse of an $\mathrm{O}+\mathrm{Ne}+\mathrm{Mg}$ white dwarf would result

Since typicul accretinn rate for calaclysmic variables is rather low $\left(\leftarrow 10^{\mathrm{A}} \mathrm{A} / \mathrm{O}\right.$ $y^{-1}$ ), it seem: unlikely that the white dwners in catnclysmic variables evolve into Type I supernovae or collnpse unless $M$ changes to exered $\sim 10 " M_{\odot} y \mathrm{r}^{-1}$ or $\mathrm{A}$ is alrendy very close to the Chandrasekhar minss.

\section{MODELS FOR SUPERNOVAE OF TYRE In AND I}

3.1 Type In Supernovac

As discussed in 52, for relatively high aecretion rates $\left(2.7 \times 10^{\circ} M_{6} y \mathrm{y}^{1}>\mathrm{M}\right.$ $\left.>1 \times 10^{*} \Lambda f_{\odot} y^{-1}\right)$, a carben defingration starts in the white dwarf's center at a relatively low central dennity $\left(\rho_{\mathrm{c}} \sim 3 \times 10^{\circ} \mathrm{g} \mathrm{cm}^{-3}\right)$ (c.s. lvanova et al. 1874; Nomoto et al. 1984 and references therein). The conmecture deflapration wave then propagates outward at subsonic velocity. The density the wave enrounters is dectcasing due to the expansion of the white dwart.

The products of explosive nucleosynthesis depend on the tempernture nnd density at the deflagration front and, thus, vary from lnyer to lnyes. In the eenter, iron pent

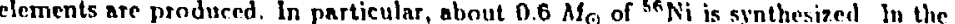
outer layers, intermedinte mass elements such as $\mathrm{Ca}, \mathrm{Ar} S$, Si ore produced The et nl. 1084; Thielemann et al. 1986; Woosley et nl. 1984). 
The carbon deflagration model enn account for the light curves, enrly time sperth, and late time spectrn of Type la supernovae as follows (see Nomoto 1980n; Woosley and Weaver 1980b for reviews and references therein):

(1) The theoretical light curve based on the ractinartive decays of ${ }^{56} \mathrm{Ni}$ and ${ }^{5 i} \mathrm{C}(\mathrm{O}$ into ${ }^{56} \mathrm{Fe}$ fits the observations well.

(2) The synthetic specturn at maximum light is in excellent ngrecment with the obscrved spectum of SN 1981b as seen in Fig. B (Branch et al. 1985; Wheeler and Ilnrkness 1986).

(3) At late times, the outer lnyers are transparent and the inner Ni-Go-Fe core is exposed. Synthetic spectra of ernission lines of $\mid \mathrm{Fe} \mathrm{II}$ and [Co I/ agree quite wel] with the spectra observed at such phase (Woosley et al. 1984).

\subsection{Type lb Supernove}

Recent observations has established the existence of nuother kind of Type I supernovere, designated Type Ib (SN Ib) (e.g., Whecler and Hnrkness 1086 and geferences therein). The SN lb splectra are characterized by the lack of the 0125 A Si feature at maximum light and the appearnnce of oxygen emission lines at late times (c.g., Gaskel et al. 1986). The currently popular progenitor models for SN Ib are Wolf-Rayet stars (e.g. Wheeler and Levreault 1085; Degelman and Sarnzin 1980). Itowever, a large mass of Wolf-Rayet stars may yaeld a light curve whose derline is too slow to be compatible with SN Ib olservations (Whecler and Levrerault 1985).
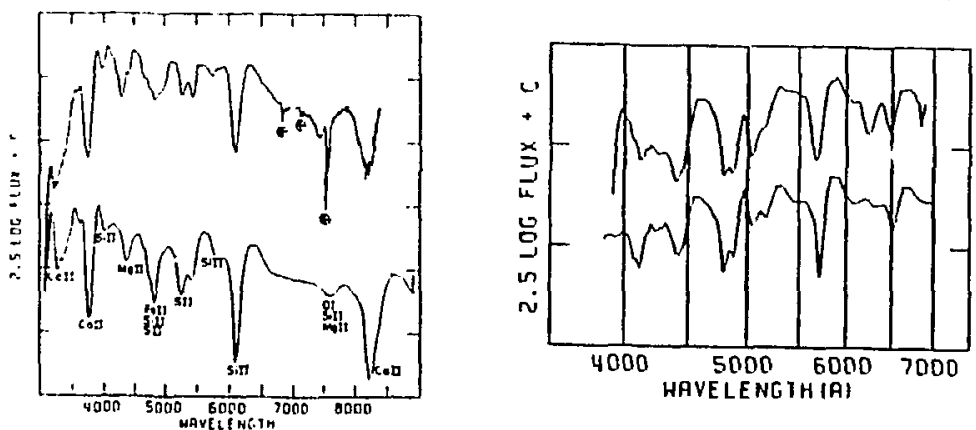

Figure 8 (left): The maximum-light spectrum of Type la SN $1981 \mathrm{~b}$ (top) is compared to a synthetic spectrum for the carbon deflagration model (Nomoto et al 1984; Branch et al. 1985). In this model outer layer is assumed to be mixed.

Figure 9 (right): The maximum-light spectrum of the Type Ib SN 1884 (upper Wheeler and Levreault 1985) is compared with a synthetic spectrum (lower; Branch and Nomoto 1986). In the synthetic spectrum the blueslifted absorption component of the I $\lambda 6678$ appears near $6500 \AA$ and 110 i $\lambda 5876$ appears near $5850 \AA$. Other fentures are produced primnrily by fo il lines.
Hinuch and Nomoto (1980) have kuggested that thr observed sperctrn nse better exBrnuch and Nomnto (1980) line kuggested that the the mined by a plained by an accreting white dwnrf nodel. In Fig. 9, the maximum-l ght spectrum of $S N 1984$ is compared with a synthetic spectrum. Two of the ahsorpition lines
in the red are identificd as He I lines and other features are well ecpplained as Fe II lines. Here the expansion velocity at the photosphere is $8,000 \mathrm{~km} \mathrm{~s}^{-1}$. In addition, ultraviolet features can fit with a synthetic spertrum of (Co II and Fe I lines if the photoshperic velocity is $12,000 \mathrm{~km} \mathrm{~s}^{-1}$ (Branch and Venkatakrishina 1980). The above interpretation suggests that Fe, Co (dernying), and He are in the outer high-velocity layers and oxygen is in the inner inyers.

The existence of such high velocity Fe nnd, especinlly, Co is diffirult to explain with the Wolf-Rayet model. Branch and Nomoto (1980) have spreculated that the progenitors of $\mathrm{SN}$ Ih are white dwarfs having $\Lambda<<4 \times 10^{A} \Lambda_{\odot} \mathrm{yr}^{-1}$. Such n slow acrection induces an off-rentes helium detonntion that will ocrur at a point ratlier than all over a spherical shell (Fig. 5). The outer helium layer will burn to mostly ${ }^{B 6} \mathrm{Ni}$ with a trace lle and the inner $\mathrm{C} / \mathrm{O}$ eore will remain unhurned. A part of most of the $\mathrm{C}+\mathrm{O}$ will be ejected following ${ }^{k 6} \mathrm{Ni}$ lnyer. Since the ejected mass of ${ }^{56} \mathrm{Ni}$ will be ne small as $0.1-0.3 M_{\odot}$, the neak luminosity of this model is lowser than that ior $5 N$ la by a ractor of $2-O$. This is colsistent with the alserations Sha hy a fincto whited a $\left.-10^{-7} M_{E}, y^{-1}\right)$ of a relatively mnssive $\left(t-i M_{\infty}\right)$ red giant companion (lben and Tutukov 1064), the model would be consistent with relatively young nature of SN Ih. Further, this scennrio ir consistent witl, the radin observations of SN Ib in that they can le explained by the internction of supernova ejocta with the eireumstellar shell (Sramek et al. 1984; Chevalier 1984).

If this speculation is correct, the precursor systoms of Type lb supernovac would he symbiotic stars rather than cataclysmic varialiles.

3.3 Dim Type I Supernovae

If an off-center single detonation occurs on a very massive white dwarf $(x \sim 1.1$ $\left.\mathbf{M}_{\odot}\right)$, the resulting supernova will be rather dim, herause the arcumulation of only a small amount of helium $\left(\sim 0.01-0.1 M_{\odot}\right)$ ean learl to the helium detonation (Fig. 5). In most cases, an unburned $C+O$ core will be left hehind as a white $d w$ nrf. Such dim supernovare (Branch and Doggett 1985) are more likely to be associated with $\mathrm{O}+\mathrm{Ne}+\mathrm{Mg}_{\mathrm{g}}$ white dwarfs since their masses are larger than $\sim 1.2 \mathrm{M}_{\odot}$

\section{NEUTRON STAR FORMATION FROM ACCRETION-INDUCED} COLLPASE OF WHITE DWARFS

Possible models for the white dwarf collapse involve solid $\mathrm{C}+\mathrm{O}$ white dwarfs, in which earhon and oxyen may or may not have chemienlly separnted (Canal et al. 1980; lsern et al. 1983) and O+Ne+Mg white dwarfs (Nonoto et al. 1979) 
4.1 C. O White Dwarfs

The $C+O$ white dwarfs could ejther explede or collnpse, depending on the conditions of the white dwarfs and binnry systems in which they are formed. Chemicat spparation in surh objects is still hypothetical and its timescale is not acrurately knuwn. It takes a carbon fraction of only a few pereent to sustain a deflagration. known. It takes a carton fiaction of only a few pesecnt to sustain a defagration.

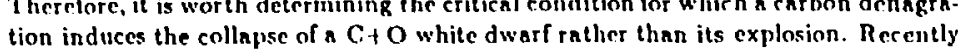
Nomoto (1986h,c) has examined such a critical condition. If a carlon deflagratin is initinted in the center of the white dwarf when $f_{\mathrm{c}} \simeq 10^{10} \mathrm{~g} \mathrm{~cm}^{-3}$ and if the props gation velocity of the deflagration wave is slower than a certain critical speed, verit, the outcome is collapse, not explosion, as seen in Figs. 10 - 11 (Nomoto 1986h, sue also lsern et at. 1084). For vdef $>v_{\text {crit }}$ Complete disruption iesults (and the carton ignition. For $\rho_{c}=1 \times 10^{10} \mathrm{~g} \mathrm{~cm}^{-3}$, "crit $\sim 0.15$ ". where $v_{\mathrm{g}}$ is the sound sperd. A lower $p_{c}$ implies a lower verit. In our case of $p_{c}=1 \times 10^{10} \mathrm{~g} \mathrm{~cm}^{3}$, firr hoth conductive and convective deflagrations $v_{\text {'del }}<v_{\text {crit }}$ and, therefore, collpase will resuit (sce Nomoto 1986b,c for more details).

Surlt a high central density is reached in two regions of the $M-M_{\text {Co }}$ plane of Fig. 6. One is defined by $M / 4 \times 10^{-8} M_{\odot} y^{-1}$ and $M f_{\mathrm{CO}}>1.1 M N_{\odot}$, while the other is defined by $\Lambda<10^{-9} M_{\odot} y \mathrm{r}^{-1}$ and $M_{\mathrm{CO}}>1.13 \mathrm{M}_{\odot}$. The frequency of

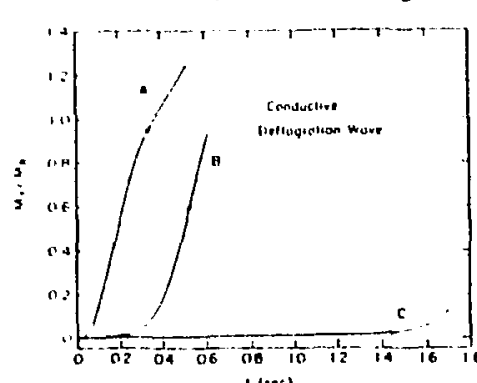

(1)es

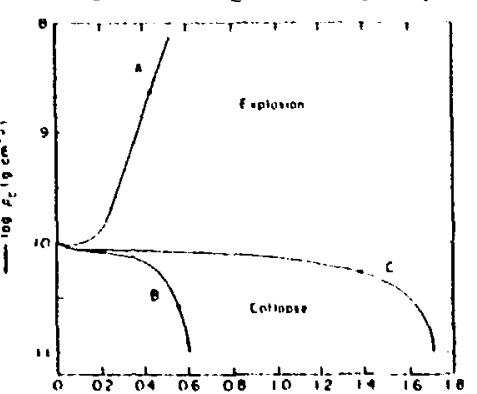

Fipure 10 (left): Propagation of the conductive deflagration wave in the $\mathrm{C}+\mathrm{O}$ white Figure 10 (left): Propagation of the conductive detagration wave in the $\mathrm{C}+\mathrm{O}$ white
dwarf. The location $\left(M_{r}\right)$ of the defingration front is shown as a function of time, $t$. for three cases $(A, B, C)$ of parametrized conductivity.

Figure 11 (right): Change in the central density of the $C+O$ white dwarf associnted with the propagation of the conductive deflagration wave. Relatively slow propatotion in Cases $B$ and $C$ leads to the inerense in pe i.e collapse of the white dwarf. On the other hand, faster pronpegation in Case $A$ induces the explusion of the white such systems may he stunll. First, for necretion at $\dot{M}<10^{-0} \mathrm{M}_{\mathcal{O}} \mathrm{yr}^{-1}$, a nova-like explosion or helium detonation will prevent the white dwarf mase from growing. Secondly, massive $\mathrm{C}+\mathrm{O}$ white dwarfs $\left(>1.2 M_{\odot}\right)$ may be rare. The formation of such white dwarfs might be prevented if the prerursor atar lust its hydrogen-rich envelope by either a stellar wind or Rnclir-lobe overflow before its degenerate $\mathrm{C}+\mathrm{O}$ core could grow substantinlly.

$4.2 \mathrm{O}+\mathrm{Ne}+\mathrm{Mg}$ White Dwarfs

As aren in Fig. 7, the accretion-induced collapse is the outeome for a wider range of parameter space for $O+\mathrm{Ne}+\mathrm{Mg}$ white dwarfs. The triggesing mechnnism of the collapse is electron capture on ${ }^{24} \mathrm{Mg}_{\mathrm{g}}$ and ${ }^{20} \mathrm{Ne}$ (Nomoto et al. 1979; Miyaji et al. 1980). The initial mass of the white dwarf, $M I_{O N}$. (Nomoto 1980, 1984a). In many cases, $M M_{O N+M g}$ is very close to the Chandrasekling
(N) (Nomoto 1980, 1984a). In many cases, $M_{O N+N}$ is very close to the Chaudlasecklons

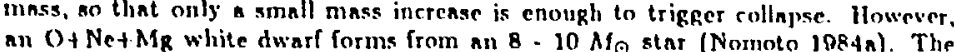
an $O+$ Net $M_{R}$ white dwarf forms from an $8-10 \lambda f_{Q}$ star (Nornoto 1084n). The
number of surh aystems nany be significantly smaller than the number of system containing $\mathrm{C}+\mathrm{O}$ white dwarfs whose precursors are 1 - $8 M / \infty$ stars, perlians, hy four order of mngnitude (lleen and Tutukov 1984). Even so, the number of low mass $X$-ray binaries is murh smaller than the number of Type 1 supernovne nnd the statistics may be consistent (Webbink et al. 1983).

\section{HELIUM STAR CATACLYSMICS}

5.1 Mnss Trnusfer from a Helium Main-Sequence Star due to Gravitational Wave Radiation

In recent studies of possible crolutionary paths of close binary stars, Tornambì and Matteucri (1886) and then and Tutukov (1086) have found that, for a relatively large range of initial parameters, systems may evolve into a confipuration consisting of a more massive $C+O$ dwarf and a less massive helium star close enough that mase transfer from the helium star to the degenerate dwarf ean be driven by gravitationa wave radiation (GWR). If GWR is the only soure of angular momentum loss, accretion rates $\Lambda f_{11}$ expected for such hehum star catarlysmes can be approxinated by (Ihen et al. 1986)

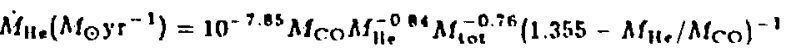

where $M_{C O}$ and $M_{11}$ are the masses (in solar units) of the accreting object and of the donor, respectively, and $M_{\text {tos }}=\Lambda I_{C O}+M_{H_{e}}$ In Fig 12 we plot curves of constant $\dot{M}_{11}$. that follow from equation (1). The region bounded by the dash-dot curve defines where initial component masses are predicted to lic (lben and Tutukov 1986). Several evolutionary tracks are shown in Fig. 12 (lines A.B.C. and D). Here the coordinate $A I_{C O}$ is to be interpreted as the sum of tic initial mass of the $C+O$ 
Wwarl and the mass $\Delta M_{H e}$ of nccreted helium.

We note first that, for all permissible initial masses, $M_{\mathrm{He}}<4 \times 10^{-\mathrm{B}} M_{\odot} \mathrm{yr}^{-1}$ Therefore, for initial masses in, say, the upper half of the sllowed region, and for systems in which the abundance of $14 \mathrm{~N}$ is low, a helium detonntion will terminate

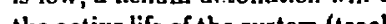
the active life of the system (track $A$ ). The
resulting explosion would look-like a Type lb supernova (see $§ 3.2)$.

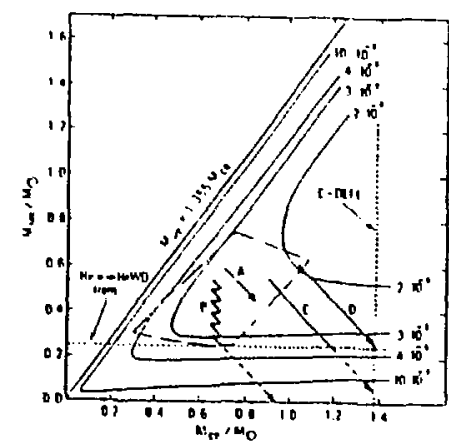

Figure 12
5.2 Mass Transfer after Helium Burning is Quenched

For systems with initial masses in the lower portion of the allowed region, scveral additional faetors must be taken into account. When its mass decreases below some minimum value $M_{\min } \sim 0.28-0.3 M_{O}$ a helium star can no longer burn helium and it will attempt to evolve into a degenerate dwarf. Evolutionary calculations (Savonije et al. 1986; Nomoto et al. 1986) have shown that, as $M_{\text {He }}$ decreases below $\boldsymbol{M}_{\min }$ and helium burning goes out, the timescalc of conling and contraction of the helium star becomes much larger than the orbital decay timescale $\left(r_{\mathrm{G}} \mathrm{w}\right.$ ). As a result, the radius of the helium star is sraaller than the value given by the mass-radius relationship used to obtain equation (1) but significantly larger than that of a fully degenerate configuration. This prevents the donor from detatching that of a rely degher

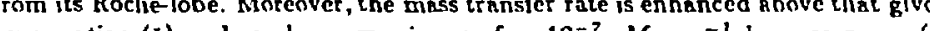
by equation (1) and reaches $a$ maximum of $\sim 10^{-7} M_{\odot} y_{\mathrm{r}}^{-1}$ because $r_{\mathrm{GW}}$ (o $\left.\Lambda \mathrm{He}^{-1}\right)$ is proportional to the fourth power of the radius. When the star hecomes sufficiently degenerate, its radius starts to increase as its mass decreases. Then the mass aceretion rate declines monotonically as $\tau_{\mathrm{G}} \mathrm{W}$ increases.

Thus, thermal inertia appears to have the effect of considerably enhaneing the mass transfer rate above $\dot{M}_{\text {det }}$ for $M_{1 l_{e}}<M_{\text {min }}$. The resulting enhaneed heating of the accreted helium layer will ignite a mild helium flash, not a detonation, unless the helium layer has already been ton massive io avoid the detonation. Such a system will end up as a $\mathrm{C}+\mathrm{O}$ white $d$ warf becauge total mass of the system is smaller than $1.38 M_{C}$ in most cases.

\subsection{The Hole of the NCO Reaction and Additional Angular Mornentum Los}

The picture is altered if we invoke an additional source of angulnr innmentum loss at a strength comparable to the GWR source and/or a suficient abundance of " $\mathrm{N}$ in the accreted helium for the efficient ${ }^{14} \mathrm{~N}\left(\mathrm{e}^{-}, \nu\right)^{14} \mathrm{C}(\alpha, \gamma)^{18} \mathrm{O}$ reaction (Hashimoto et al. 1986). Now $\dot{M}_{H_{e}}>\dot{M}_{\text {det }}$ as far as $M_{\mathrm{He}}>\sim 0.1 M_{\odot}$ so that all models which have initial masses in the region suggested by binary scenarios will experience relntively mild flashes. Several untested possibilities for evolutionary paths arise. If the mass acereted betwcen two successive flashes is converted into carbon and oxygen that remsins on the acereting star, then there exists a region in initial parameter apace such that the underlying $\mathrm{CO}$ core will grow until it reaches the critical value of $1.38 M_{\odot}$, at which point a carbon deflngration will be ignited. In other words, Type la supernovae would be the possible outcome of the crolution starting from the upper-right of track $\mathrm{C}$ in Fig. 12 where $M_{H_{\mathrm{e}}}+M_{\mathrm{CO}}>1.48 M_{\mathrm{O}}$.

5.4 Helium Star - O+Ne+Mg White Dwarf Fair and the Origin of

Ultrashort Period X-Ray Binaries

If the helium star has an $\mathrm{O}+\mathrm{Ne}+\mathrm{Mg}$ white dwarf companion, the initinl mass of the white dwarf is larger than $\sim 1.1 M_{\odot}$, even as large as $\sim 1.36 M_{\odot}(\S 6)$. Therefore, for a wider range of initial parameters in Fig. 12, the $\mathrm{O}+\mathrm{Ne}+\mathrm{Mg}$ white dwarf evolyes to collapse if $\dot{M}>\dot{M}$. $<0.3 M_{0}$ even if GWR is the only source of angular momentum loss, and for a much wider range of initial mass if the additional effects operate as discussed in 55.3. Then the system would become an ultrashor: period X-ray binary system such as $\mathrm{X}$-rny pulsar $1 E 2259+586$ whose orbital period is $38 \mathrm{~min}$ (see Savonije et al. 1986 for more details). Accretion-induced collapse in helium star ealaclysmics would not occur for $C+O$ white dwarfs if the initial mnss is hounded as in Fig 12 (Then and Tutukov 1986) because $M_{\mathrm{CO}}>\sim 1.2 M_{\odot}$ is required for the collapse to occur (Fig. 6).

\subsection{S - Process Nucleosynthesis}

If $\dot{M}<\dot{M}$ and a large fraction of the matter accreted between pulses is rjected during pulses, then, since s-process isotopes are formed in the convective shell during pulses (Iben 1981; Fujimoto and Sugimoto 1982), heliurn star cataclysmics may be important sources of G-process isotopes (Bben and Tutukov 1986). If the NCO reaction operates, the neutrons are produced by the ${ }^{14} \mathrm{~N}\left(\mathrm{c}^{-}, \nu\right)^{14} \mathrm{C}(\alpha, \gamma)^{18} \mathrm{O}(\alpha, \gamma)$ ${ }^{22} \mathrm{Ne}(\alpha, \pi){ }^{25} \mathrm{Mg}$ reactions. If a significart fraction of the material processed by helium fosher is not ejeted during pulses, a Type la supernova will occur and eject 5-process elements which remain in the outer unburned layers (Nomoto et al. 1984). 


\section{NEON NOVAE AND COMPOSITION OF O+Ne+Mg WHITE DWARFS}

\subsection{Neon Novae}

The evolution of $\mathrm{O}+\mathrm{Ne}+\mathrm{Mg}$ white dwarfs hes been discussed in the context of white dwarf collapse assuming their formation from certain stars (Finzi and Wolf 1967; Nomoto et al. 1979). The actual stellar evolution through the formation of $\mathrm{O}+\mathrm{Ne}+\mathrm{Mg}$ white dwarfs has been shown in the calculation of helium stars by Nomoto $(1980,1981,1984 a, b)$. Recently the $\mathrm{O}+\mathrm{Ne}+\mathrm{Mg}$ white dwarf lias attracted another attention because some novae show enrichment of $\mathrm{Ne}, \mathrm{Mg}, \mathrm{Na}$, and $\mathrm{Al}$ in the ejecta (e.g., Williams et al. 1985; Gehrz et al. 1985). One of the possible models is that the neon novae occur on the $\mathrm{O}+\mathrm{Ne}+\mathrm{Mg}$ while dwarfs (Law and Ritter 1984; Delbourgo-Salvador et al. 1985; Starrfield et al. 1986; Truran and Livio 1986). This model invokes some mixing between the accreted matter and the underlying core matcrial so that the composition of the cjectn must reflect the abundance of the $\mathrm{O}+\mathrm{Ne}+\mathrm{Mr}$ core. To confirm this idea, we have to know the original composition structure of the $\mathrm{O}+\mathrm{Ne}+\mathrm{Mg}$ white dwarf and compare the theoretical prediction of nucleosynthesis with the observed abundance. Regarding the composition, recently inflated ${ }^{12} \mathrm{C}(\alpha, \gamma){ }^{16} \mathrm{O}$ reaction rate changes nucleosynthesis during the course of stellar evoultion. We report below our new evolutionary calculation for the formation of the $\mathrm{O}+\mathrm{Ne}+\mathrm{Mg}$ white dwarfs (Nomoto and Heshimoto 1986).

6.2 Evolution of Helium Stars in Close Binaries through Formation of $\mathrm{O}+\mathrm{Ne}+\mathrm{Mg}$ White Dwaris

If one of the component star of a close binary is a $8-13 M 1_{0}$ star, it becomes a helium star of mass $M_{0} \sim 2-3.3 M_{0}$ after tidal mass loss. The helium star in this mass range undergoes non-degenerate carbon burning and forms a semi-clegencrate $\mathrm{O}+\mathrm{Ne}+\mathrm{Mg}_{\mathrm{g}}$ core (Nomoto 1984a; Habets 1985, 1986). For $2.8<M_{\mathrm{p}}<3.2 M_{\odot}$, neen burning is ignited off-center and the neon-oxygen burning front propagates inward all the way to the center. Subsequent evolution forms an iron core (llashimoto and Nomoto 1986; see Nomoto and Hashimoto 1086a). For $M_{\mathrm{a}}<2.8 M_{\odot}$, the advance of the carbon burning thell (i.e. Browth of the $0+\mathrm{Ne}+\mathrm{Mg}$ core) stops near the $M_{\odot}$. respectively. Hence the $\mathrm{O}+\mathrm{Ne}+\mathrm{Mg}$ corc mass does not exceed the critical mas of $1.37 M_{\odot}$ for neon ignition and, thus, neon burning is never ignited. Afterwards, the core becomes strongly degenerate. The radius of the helium star increnses as the $\mathrm{O}+\mathrm{Ne}+\mathrm{Mg}$ core grows and reaches as large as $\sim 1000 R_{\odot}$ if it is a single star. In a close binary system, certainly Roche lobe overflow occurs and an $O+N e+M$ white dwarf of mass $M_{\mathrm{wd}} \simeq M_{\mathrm{Heb}}$ is left.

\subsection{Composition Structure of $\mathrm{O}+\mathrm{Ne}+\mathrm{Mg}$ White Dwarf}

In Figs. $13-14$, the composition structures of helium stars of $M_{a}=2.6$ and 2.7 $M_{\odot}$ nenr the onset of Roche lobe overflow are shown. After the heliurn envelope i lost, the dwarf residue consists of an $\mathrm{O}+\mathrm{Ne}+\mathrm{H} / \mathrm{g}$ corc and an outer $\mathrm{C}+\mathrm{O}$ layer. The $\mathrm{C}+\mathrm{O}$ layer is thinner for lager white dwarf mass because the density at the bottom of the $\mathrm{C}+\mathrm{O}$ layer is $\sim 1.5 \times 10^{5} \mathrm{~g} \mathrm{~cm}^{-3}$ and a mass of overlying layer is smaller for larger mass white dwar's which have smaller radius. For example, $\Delta M A_{C+O}=$ 0.036 and $0.005 M_{\odot}$ for $M_{W D}=1.27$ and $1.36 M_{\odot}$, respectively. This implies that a larger $\mathrm{C}+\mathrm{O}$ lnyer must be eroded by accretion and outburst until neon-rich layer is exposed (Truran and Livio 1986).

The abundances taken from Figs. 13 - 11 are summarized as follows. For $M_{\alpha}=2.7$ $M_{\odot}\left(M_{W D}=1.36 M_{\odot}\right)$, mass fractions are $X(C)=0.30, X(0)=0.66, X(\mathrm{Ne})$ $=0.018$ in the $\mathrm{C}+0$ layer. For the core at $M_{\mathrm{r}}=1.34 M_{\odot}, X(0)=0.54, X(\mathrm{Nc})$ $=0.37, X(\mathrm{Na})=0.013, X(\mathrm{Mg})=0.049, X(\mathrm{Al})=0.01$, and $X(\mathrm{Si})=0.003$. The abundances of the $1.20 M_{\odot}$ white dwarf are similar. Compareu with the old models (Nomoto 1984a), the $\mathrm{C} / \mathrm{O}$ satio in the $\mathrm{C}+\mathrm{O}$ inyer and the $\mathrm{Ne} / \mathrm{O}, \mathrm{Mg} / \mathrm{O}$ rntios in the core are significantly smaller in new models becnuse of the larger ${ }^{12} \mathrm{C}$ $(\alpha, \gamma)^{16} \mathrm{O}$ reaction rate. If the $\mathrm{O}$-Ne-rich core meterial cons iitutes $20-30$ percent of the nova ejecta, the abundance of the $\mathrm{O}+\mathrm{Ne}+\mathrm{Mg}$ white dwarf is consistent with the observed abundance of Nova CrA 1981 (Williams et al. 1985)

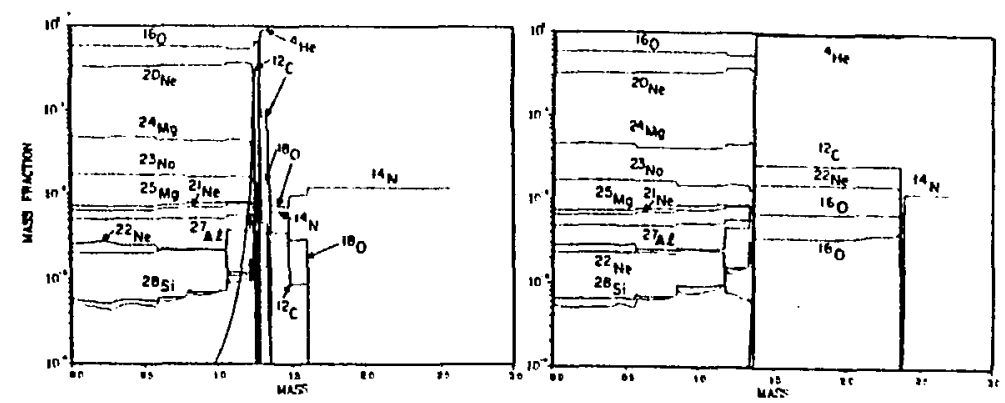

Figures 13 - 14: Composition of the heliurn ktars of $M_{0}=2.6 M_{0}$ (left) and 2.7 $M_{\odot}$ (right). After the Roche lobe cvedfow, $O+N_{e}+M_{F}$ whito dwarfe of massere 1.97 
Another characteristic of the $\mathrm{O}+\mathrm{Ne}+\mathrm{Mg}$ white dwarfs compared with $\mathrm{C}+\mathrm{O}$ white dwarfs is a significantly larger mass $\left(>\sim 1.1 \Lambda_{\odot}\right)$, even close to the Chandrasckhar unass, and, hence, smaller radius. The lower mass limit of $\mathrm{O}$ Ne $\mathrm{Ng}$ white dwarfs is somewhat Inrger then $1.00 \mathbf{M}_{\odot}$ below which no earbon ignition oceur, hecause the core mass still increnses after carbon ignition until the Roche lobe overflow commences. The upper mass limit is $1.37 \mathrm{M}_{\odot}$ nhove which neon is ignited (Nomoto 1984a). Because of the large white dwarf mass, $\Delta M_{\| 1}$ at the ignition of hydrogen nash is as small as $10^{-6}-10^{-b} M_{\odot}$ (Fig. 2) so that the ejecta mass is small (Starrficld et al. 1980).

\section{CONCLUDING REMARKS}

Though there is a gond agreement between the exploding white dwarf models and the observed spectra and light curves of Type la supernovae, the exact precursor systems where the white dwarf mass grows to the Chandrasekhar mass are not systems where the white dwarf mass grows to the Chandrasekhar mass are not
know yet. Typical cataclysmic variables seem not to be the promising candidntes of kunew yet. Typical cataclysmic variables seem not to be the pronising candidntes of kupernova precursors hecause mass accretion rate of the catnclysmic variables is too low to increase the white dwarf mass up to the Chandrasekhar mass. However, re lated systems such as double white dwarfs, helium star cataclysmics, and symbiotic
stars deserve further exploration.

Recent break through in supernova studies has been brought about by the calruIntions of theoretical spectrn at various stnges of supernova outburst which are in good agreement with the observed spectra ( $\$ 3)$. For this purpose, rndiation hydro cocte has been developed by several groups. If the snme approach is possible for cote has been developed by several groups. If thic same approach is possible for nova studies by calculating model atomospheres and synthetic specta, it would certainly be quite fruitful. Besides rndiation hydrodynamics, detailed nucleosynthesis calculation based on realistic $\mathrm{O}+\mathrm{Ne}+\mathrm{Mg}$ white dwarf snodels are needed for the
st udy of neon novae.

Wi would like to thank Drs. 1. Iben, A. Tornambe, and H. Snio for stimulating discussion on helium star cataclystnics and merging white dwarfs. It is pleasure to thank Drs. S.H. Kahana, G.E. Brown, A. Yahil, A. Burrows, J. Cooperstein, and E. Baron for useful discussion and the hospitality during our stay in Brookhaven nnd Stony Brook. This work has been supported in part by the $U$. S. Department of Energy under Contract No. DE-ACO2-76 of Education, Science, and Culture through of Education, Science, and Culture through research grant Nos. 59380001 and

\section{REFERENCES}

Begelman, M.C., and Sarazin, C.L. 1986, Ap. J. (Letters), 302, L50. Branch, D., and Doggett, 3.B. 1985, A. J., 270, 2218

Branch, D., Doggett, J.B., Nomoto, K, and Thielemann, F.-K. 1085, Ap. J., 294, 610

Brauch, D., and Nomoto, K. 1986, Astr. Ap., in press.

Branch, D., and Venkatakrishna, K.L. 1086 Ap. J. (Letters), 306, L21

Canal, R., Isern, J., and Labny, J. 1980, Ap. J. (Letters), 241, L33.

Chevalier, R.A. 1984, Ap. J. (Letters), 285, L63.

Delbourgo-Salvador, P., Mochkovitch, and Vangioni-FInrn, E. 1985, in Recent Results on Cataclysmic Variabies (ESA SP-230), p. 220

Finzi, A., and Wolf, R.A. 1967, Ap. . I, 150, 115

Fujimoto, M.Y., and Tanm, R.E. 1982, Ap. J., 260, 219

Fujimoto, M.Y., and Sugimoto, D 1982, Ap. J., 257, 291.

Gaskell, C.M., Gappellaro, E., Dinerste in, H., Garnett, D., Harkness, R.P., and Wheler, J.C. 1086, Ap. I. (Letters), 306, L77.

Gehrz, R.D., Grasialen, G.L., and Hackwell, J.A. 1985, Ap. J. (Letlers), 298, L47. Habets, G.M.H.J. 1985, Ph.D. Thesis, University of Ansterdam.

- 1986, Astr. Ap., submitter.

IInshimnto, M., and Nomoto, K. 1986, in preparation.

Hashimoto, M., Nomoto, K., Arni, K., and Kaminisi, K. 1986, Ap. J., 307, 087 Iben, I. Jr. 1981, Ap. .I., 243, 087.

Iben, I. Ir., Nomoto, K., Tornambè, A, nnd Tutukov, A.V. 1986, Ap. J., submitted.

Iben, I. Jr., and Tutukov, A.V. 1984, Ap. J. Suppl., 54, 335

1986, Ap. J., submitted

Iscrn, J Labay, J., Hernamz, $M$ and Canal, $R$, 1983, Ap. J., 273, 320

Isern, J., Lahay, J., and Canal, R. 1984, Nature, 309, 431.

Jvanova, L.N., Imshennik, V.S., and Chechetkin, V.M. 1974, Ap. Space Sci., 31, 407.

Kawni, Y., Saio. H., and Nomoto, K. 1986, Ap. J., submitted.

Law, W.Y., nnd Ritter, II. 1983, Astr. Ap., 123, 33.

MacDonald, J. 1083, Ap. J., 26:, 732

-

Miyaji, S., Nomoto, K., Yokoi, K., and Sugimoto, D. 1980, Pub. Astr. Soc. Japan, 32,303

Nariai, K., and Nomoto, K. in IAU Colloquium 53, White Dwarfs and Variable Drgenernte Stars, ed. H.M. Van Horn and V. Weidemann (Rochester: Univ. of Rochester), p.525.

Nomnto, K. 1980, in Type I Supernovae, ed. J. C. Whecler (Austin: University of Texas), p. 104.

- 1881, in IAU Symposium 93, Fundamental Problems in the Theory of Stellar Evolution, ed. D. Sugimoto et al. (Dordrecht: Reidel), p.295. 
Nomoto, K. 1982n, Ap. I., 253, 798.

1982b, Ap. J., 257, 780

$1084 a$, Ap. J., 277, 791

1084b, in Problems of Collapse nnd Numeriral Reintivity, ed. D. Bnneel

and M. Signore (Dordrecht: Reidel), p.80.

1986a, Ann. N Y Aead. Sei., 470, 294

1986a, Ann. N Y Acad. Sri, 470, 294 . Astrophysics Mecting: Accretion

Processes in Astrophysics, in press.

. 1086c, in IAU Symposium 125, The Origin and Evolution of Neutron

Stars, ed. D.J. Helfand and J.H. Huang (Dordrecht: Reidel), in press.

Nomoto, K., and Hashimoto, M. 1980, Prog. Pnrt. Nucl. Fhys., 18, in press.

Nomoto, K., Hashimoto, M., Jben, I. Jr., and Tornambè, A. 1986, in preparation.

Nomnto, K., and Iben, I. Ir. 1985, Ap. J., 297, 531.

Nomoto, K., Miyaji, S., Yokoi, K., and Sugimoto, D. 1970, in IAU Colloquium 53,

White Dwarfs and Variable Degenerate Stars, ed. H.M. Van Horn and

V. Weidemann (Rochester: Univ, of Rochester), p.56.

Nomoto, K., and Sugimoto, D. 1977, Pub. Astr. Soc. Japan, 29, 765.

Nonnoto, K., Thiclemann, F.K., and Yokoi, K. 1984, A p. J., 286, 644.

Saio, H., and Nomoto, K. 1985, Astr. Ap., 150, L21.

Snvonije, G.J., de Kool, M., and van den Heuvel, E.P.J. 1986, Astr. A I., 155, 51

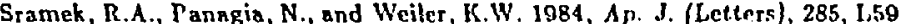

Starrfield, S., Sparks, W.M., and Truran, J.W. 1986, Ap. J. (Letters), 303, L5.

Tram, R.E. 1980, Ap. J., 237, 142

Taam, R.E., and van den Heuvel, E.P.J. 1986, Ap. I., 305, 235

Thielemann, F.-K., Nomoto, K., and Yokoi, K. 1986, Astr. Ap., 158, 17

Tornambè, A., and Matteucci, F. 1986, M.N.R.A.S., in press.

Truran, J.W., and Livio, M. 1986, Ap. J., 308, in press.

Van den Heuvel, E.P.I. 1984, J. Ap. Astr., 5, 209

Webhink, R. 1984, Ap. J., 277, 355.

Webhink, R.F. Rappmport, S., and Snvonije, G.J. 1983, Ap. J., 270, 678

Wheler, J.C., and Harkness, R. 1986, in Distanees to Gnlaxies and Deviation from the Hubble Flow, ed. B.M. Madore and R.B. Tully (Dordrecht: Reidel).

Wheeler, J.C., and Levreauli, R. 1885, Ap. J. (Letters), 294, L.17.

Williams, R.E. et al. 1985, M.N.R.A.S., 212, 753.

Wonsley, S.E., Axclrod, R.S., and Wenver, T.A. 1984, in Stellar Nucloosynthesis,

$$
\text { ed. C. Chiosi and A. Renzini, (Dordrecht: Reidel), p.203. }
$$

Woosley, S.E., Taam, R.E., and Weaver, T.A. 1986, Ap. J., 301, G01.

Wonsley. S.E., and Weaver. T.A J986n, in Nulleospnthrsis and Its lmulications

for Nuclear and Particle Plyysics, cd. J. Audouze and T. van Thuan (Dordrecht: Reidel)

- 1986b, Ann. Res. Astr. Ap., in press. 